\title{
Effects of Channel Estimation on Spatial Modulation
}

\author{
Shinya Sugiura, Senior Member, IEEE, and Lajos Hanzo, Fellow, IEEE
}

\begin{abstract}
In this letter, we investigate the effects of trainingbased channel estimation on the achievable performance of the recent spatial modulation (SM) based multiple-input multiple-output (MIMO) scheme. This is motivated by the fact that the SM transmitter is constituted by a single radio-frequency (RF) branch and multiple antenna elements (AEs), hence simultaneous pilot transmissions from the AEs are impossible, unlike in the classic multiple-RF MIMO transmitters. Our simulation results demonstrate that the SM scheme's BER curve exhibits a performance penalty, while relying on realistic imperfect channel-estimation. In order to combat these limitations, we propose two single-RF arrangements, namely a reduced-complexity joint channel estimation and data detection aided SM scheme as well as a non-coherently detected single-RF space-time shift keying scheme dispensing with channel estimation.
\end{abstract}

Index Terms-Channel estimation, iterative detection, non-coherent detection, single-RF MIMO, space-shift keying, space-time shift keying, spatial modulation, training signals.

\section{INTRODUCTION}

$\mathbf{T}$ HE RECENT encoding concept of spatial modulation (SM) [1]-[3] enables us to exploit the potential of multiple-input multiple-output (MIMO) channels with the aid of a single radio-frequency (RF) transmitter arrangement. More specifically, the SM scheme is characterized by a specific antenna element (AE) activation process, where the index of one out of $M$ transmit AEs conveys additional information. This implies that an increase in the transmission rate is achieved by increasing the number of transmit antennas, rather than by relying on spatial multiplexing activating all the $M$ AEs. Detailed performance comparisons of SM and of the classic MIMO systems have been carried out in [4], [5] and the references therein, while assuming that perfect knowledge of the channel state information (CSI) is available at the receiver.

By contrast, considering that the SM scheme is especially beneficial for a high number of AEs, it is of prime significance to explore the effects of pilot overhead and channel estimation errors. Specifically, the effects of channel estimation

Manuscript received July 12, 2012; revised September 01, 2012; accepted September 11, 2012. Date of publication October 02, 2012; date of current version nulldate. The work of S. Sugiura was supported in part by the Japan Society for the Promotion of Science (JSPS KAKENHI Grant 23760353). The financial support of the RC-UK under the auspices of the India-UK Advanced Technology Centre, as well as of the China-UK 4th generation wireless systems project and that of the European Union under the auspices of the Optimix project, is gratefully acknowledged. The associate editor coordinating the review of this manuscript and approving it for publication was Prof. Azadeh Vosoughi.

S. Sugiura is with Toyota Central R\&D Laboratories, Inc., Nagakute, Aichi, 480-1192, Japan (e-mail: sugiura@ieee.org).

L. Hanzo is with the School of Electronics and Computer Science, University of Southampton, Southampton, SO17 1BJ, U.K. (e-mail: 1h@ecs.soton.ac.uk).

Digital Object Identifier 10.1109/LSP.2012.2221707
(CE) errors on the SM scheme were investigated in [6], [7], by assuming that the CE error was Gaussian. Furthermore, diverse solutions, dispensing with this idealized simplifying assumption, were proposed in [8], [9], where the SM scheme was extended to support non-coherent detection in [8], while joint channel estimation and data detection was proposed in [9]. Furthermore, since the SM scheme is equipped with a single RF branch and multiple transmit AEs, it is impossible to simultaneously transmit multiple pilot symbols from the antenna elements, unlike in the classic multiple-RF MIMO transmitters. This limitation may impose a performance penalty on the coherently-detected single-RF SM scheme.

Against this background, the novel contributions of this letter are two-fold. We first investigate the performance of the coherently-detected SM scheme, while taking into account the effects of training-based channel estimation at the receiver. Additionally, the single-RF SM scheme's performance penalty is evaluated in terms of the mean-square errors (MSEs) of the estimated channels in comparison to the classic multiple-RF MIMO systems. Furthermore, we propose two single-RF MIMO arrangements, namely a reduced-complexity joint channel estimation and data detection aided SM scheme as well as the non-coherently detected space-time shift keying (STSK) scheme. It is demonstrated by our simulations that the proposed schemes have the potential of combating the above-mentioned CSI-error related limitations.

\section{Signal MODEL}

\section{A. Spatial Modulation}

Consider the transmitter, relying on $M$ transmit AEs and on classic $\mathcal{L}$-point complex-valued constellations, such as phase-shift keying (PSK) and quadrature-amplitude modulation (QAM). We assumed single-carrier transmissions, according to the related studies [1]-[5]. ${ }^{1}$.

Firstly, a total of $B=\log _{2}(M \cdot \mathcal{L})$ information bits are transmitted per symbol duration, which are then serial-to-parallel (S/P) converted to $B_{1}=\log _{2} M$ and $B_{2}=\log _{2} \mathcal{L}$ bits. Furthermore, the $B_{1}$ bits are used for activating one out of $M$ transmit AEs, while the $B_{2}$ bits are mapped to a PSK/QAM symbol $s_{l}(1 \leq l \leq \mathcal{L})$. Finally, the symbol $s_{l}$ is transmitted from the activated $\mathrm{AE}$, having the index of $m(1 \leq m \leq M)$. Hence, the normalized transmission rate $R$ of the SM scheme is formulated by $R=\log _{2}(M \cdot \mathcal{L})$, which logarithmically increases upon increasing either the number of transmit AEs $M$ or the constellation size $\mathcal{L}$.

\footnotetext{
${ }^{1}$ Typically, the SM scheme based on a single-RF transmitter does not support multi-carrier transmissions. This is because if each sub-carrier employs the antenna activation process in a multi-carrier system, simultaneous symbol transmissions from multiple antenna elements become necessary.
} 
At the $N$-antenna-aided receiver, the received signals $\mathbf{y}=$ $\left[y_{1}, \ldots, y_{N}\right]^{T} \in \mathbb{C}^{N \times 1}$ may be expressed as $\mathbf{y}=s_{l} \mathbf{h}_{m}+\mathbf{v}=$ $\mathbf{H k}_{m, l}+\mathbf{v}$, where we have

$$
\mathbf{k}_{m, l}=[\underbrace{0 \ldots, 0}_{m-1}, s_{l}, \underbrace{0 \ldots, 0}_{M-m}]^{T} .
$$

Here, $\mathbf{H}=\left[\mathbf{h}_{1}, \ldots, \mathbf{h}_{M}\right] \in \mathbb{C}^{N \times M}$ represents the complex-valued channel coefficients, while each noise element of $\mathbf{v} \in \mathbb{C}^{N \times 1}$ is the complex-valued Gaussian random variable obeying $\mathcal{C N}\left(0, N_{0}\right)$, having a zero mean and a variance of $N_{0}$. Based on the received signals $\mathbf{y}$, the estimated channels $\mathbf{H}$ and noise variance $N_{0}$, the transmitted $\mathrm{AE}$ and symbol index set $(m, l)$ is identified with the aid of the optimal reduced-complexity matched-filtering (MF) detector [10].

\section{B. Training-Based CSI Estimation}

In the coherently-detected SM scheme, the training symbols $\mathbf{P} \in \mathbb{C}^{M \times t}$ are periodically transmitted in advance of a frame of data symbols, noting that the integer value of $t$ corresponds to the pilot length. Here, $\mathbf{P}$ is assumed to satisfy the power constraint of $\operatorname{tr}\left[\mathbf{P P}^{H}\right]=t$. More specifically, the associated received training block $\mathbf{Y}_{t} \in \mathbb{C}^{N \times t}$ may be written as $\mathbf{Y}_{t}=$ $\mathbf{H P}+\mathbf{V}_{t}$, where $\mathbf{V}_{t}$ represents the associated noise components, which are complex-valued Gaussian random variables obeying $\mathcal{C N}\left(0, N_{0}\right)$. Importantly, in the single-RF SM transmitter the pilot matrix $\mathbf{P}$ is sparse, where each column of $\mathbf{P}$ has only a single non-zero element, unlike in the classic multiple-RF MIMO systems.

Let us consider the linear $\mathrm{CE}$, which minimizes the estimation MSE of $\mathbf{H}$. Such a minimum MSE (MMSE) CE may be formulated by

$$
\hat{\mathbf{H}}=\mathbf{Y W},
$$

where $\mathbf{W} \in \mathbb{C}^{t \times M}$ represents the MMSE CE's weights, which are given by [11]

$$
\mathbf{W}=\left(\mathbf{P}^{H} \mathbf{R}_{\mathrm{H}} \mathbf{P}+N_{0} N \mathbf{I}\right)^{-1} \mathbf{P}^{H} \mathbf{R}_{\mathrm{H}} .
$$

Here, the channel's correlation matrix is $\mathbf{R}_{\mathrm{H}}=\mathbb{E}\left[\mathbf{H}^{H} \mathbf{H}\right] \epsilon$ $\mathbb{C}^{M \times M}$ and $\mathbb{E}[\cdot]$ is the expectation operation. Furthermore, the associated theoretical CE MSE value $J_{\mathrm{MMSE}}$ is formulated as [11]

$$
\begin{aligned}
J_{\mathrm{MMSE}} & =\operatorname{tr}\left[\mathbb{E}\left\{(\mathbf{H}-\hat{\mathbf{H}})(\mathbf{H}-\hat{\mathbf{H}})^{H}\right\}\right] \\
& =\operatorname{tr}\left[\left(\mathbf{R}_{\mathrm{H}}^{-1}+\frac{1}{N_{0} N} \mathbf{P P}^{H}\right)^{-1}\right] .
\end{aligned}
$$

Assuming for simplicity that the pilot symbols obey $\mathbf{P}=\mathbf{I}$ and that we have an uncorrelated scenario, i.e., $\mathbf{R}_{\mathrm{H}}=\mathbf{I}$, the MSE value of (5) may be simplified to $\bar{J}_{\text {MMSE }}=N_{0} M N / N_{0} N+1$. Furthermore, in the high-SNR limit $\left(N_{0} \rightarrow 0\right)$ the associated CE MSE value per AE becomes $\bar{J}_{\text {MMSE }} /(M \cdot N)=N_{0}$, which may result in a 3-dB performance penalty over the perfect-CSI scenario. Note that the single-RF based transmit antenna selection scheme [12] typically employs a similar pilot-transmission strategy and hence the resultant $\mathrm{CE}$ errors at the receiver are also similar. However, the antenna selection scheme requires feedback information from the receiver, while the SM scheme does not.

\section{Proposed Detection Schemes}

In this section, we introduce two beneficial schemes, which have the potential of combating the limitations associated with the above-mentioned pilot overhead and CSI estimation errors.

\section{A. Reduced-Complexity Joint Channel Estimation and Data Detection}

As mentioned in [9], the joint $\mathrm{CE}$ and data detection scheme is capable of reducing the effects of CE error, which is achieved without prohibitively increasing the pilot overhead. In order to minimize the performance degradation imposed by $\mathrm{CE}$, while reducing the decoding complexity, here we invoke the reducedcomplexity MF-based data detection combined with the MMSE CE. The detailed iterative process is formulated as follows:

i) Initial channel estimation: The initial values of the channel coefficients $\hat{\mathbf{H}}^{(0)}$ are estimated according to the MMSE criterion of (2), (3), while the iteration index is set to $\iota=0$. Furthermore, we set the received signal block as $\mathbf{Y}=\left[\mathbf{y}_{1}, \ldots, \mathbf{y}_{\tau}\right] \in \mathbb{C}^{N \times \tau}$.

ii) Data detection: A frame of $\tau$ transmitted SM symbols are detected with the aid of the reduced-complexity ML detector of [10], in order to attain the concatenated estimated symbol block of $\mathbf{S}^{(\iota)}=\left[\mathbf{k}_{1}^{(\iota)}, \ldots, \mathbf{k}_{\tau}^{(\iota)}\right] \in \mathbb{C}^{M \times \tau}$.

iii) Channel estimation: The channel estimates $\hat{\mathbf{H}}^{(\iota+1)}$ are updated based on the MMSE criterion of (2), (3), which is expressed as $\hat{\mathbf{H}}^{(\iota+1)}=\mathbf{Y}\left(\mathbf{S}^{(\iota) H} \mathbf{R}_{\mathrm{H}} \mathbf{S}^{(\iota)}+\right.$ $\left.N_{0} N \mathbf{I}\right)^{-1} \mathbf{S}^{(\iota) H} \mathbf{R}_{\mathrm{H}}$.

iv) Iteration or termination: If $\left\|\hat{\mathbf{H}}^{(\iota+1)}-\hat{\mathbf{H}}^{(\iota)}\right\|^{2} /(M \cdot N)<$ $\beta$, then terminate the iteration and output the estimated symbols $\mathbf{S}^{(\iota)}$ as well as the associated bits. Otherwise, set $\iota=\iota+1$ and goto 2). Furthermore, the termination constant $\beta$ is chosen to be sufficiently low.

Here, we note that the achievable bit-error rate (BER) performance is expected to improve at the expense of an increased decoding complexity, which linearly increases with the number of iterations.

\section{B. Non-Coherently Detected Single-RF STSK Scheme}

Additionally, in order to allow the single-RF SM arrangement to remain unaffected by CSI estimation errors, non-coherent detection is invoked in this section. To be more specific, as an extension of the SM scheme, the differentially-encoded space-time shift keying (DSTSK) scheme [8] was developed, which is capable of attaining a transmit diversity gain, while invoking non-coherent detection. Although the DSTSK transmitter was basically assumed to have multiple RF branches, in this letter we relax this constraint, by assuming that the DSTSK uses a single-RF transmitter. Let us assume that our single-RF transmitter has $M$ transmit AEs and $Q$ unitary dispersion matrices $\mathbf{B}_{q} \in \mathbb{C}^{M \times M}(q=1, \ldots, Q)$ [10]. Furthermore, we assume that each dispersion matrix has a sparse structure, where each column of $\mathbf{B}_{q}$ has a single non-zero unit-norm element. 


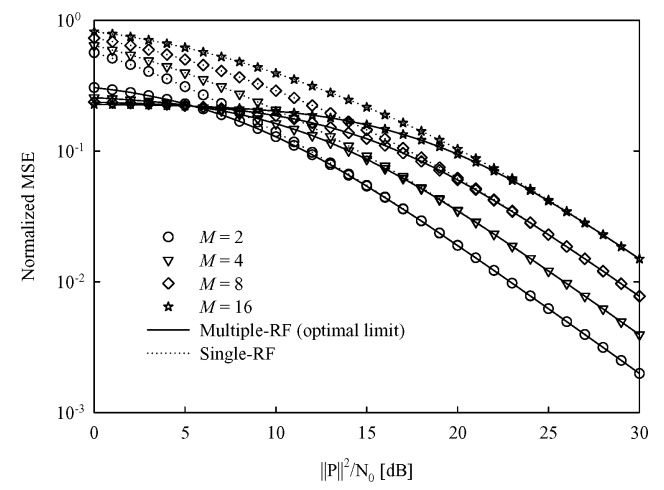

Fig. 1. Normalized MSE of the estimated channels regarding both the singleand multiple-RF pilot transmissions, where the number of transmit antennas $M$ was varied from $M=2$ to 16 , while the number of receive antennas $N$ was set to $N=4$. The antenna's correlation coefficient was given by $\epsilon=0.8$.

Similar to the SM scheme of Section II.A, the $\bar{B}=$ $\log _{2}(Q \cdot \mathcal{L})$ information bits per block are firstly $\mathrm{S} / \mathrm{P}$ converted to $\bar{B}_{1}=\log _{2} Q$ and $\bar{B}_{2}=\log _{2} \mathcal{L}$ bits, which are then signaled by activating one out of $Q$ dispersion matrices $\mathbf{B}_{q}(n)$ and by a PSK/QAM symbol $s_{l}(n)$, respectively. Here, $n$ denotes the block index. Finally, the differentially-encoded symbols are generated based on two successive received-signal blocks as follows: $\overline{\mathbf{S}}(n)=s_{l}(n) /\left|s_{l}(n-1)\right| \overline{\mathbf{S}}(n-1) \mathbf{B}_{q}(n) \in \mathbb{C}^{M \times M}$ where $\overline{\mathbf{S}}(n)$ also has a sparse matrix structure, similar to $\mathbf{B}_{q}$. This ensures that our scheme transmits a single symbol per transmission interval. The signal block received during the $n$th block interval is written as $\overline{\mathbf{Y}}(n)=\mathbf{H} \overline{\mathbf{S}}(n)+\overline{\mathbf{V}}(n) \in \mathbb{C}^{M \times M}$. Finally, the transmitted information bits are estimated without resorting to any channel estimation [10], assuming that the channel matrix $\mathbf{H}$ remains unchanged over the two block intervals. Naturally, the achievable BER performance of the single-RF DSTSK scheme proposed in this section is upper-bounded by that of the full-RF DSTSK scheme of [8].

\section{Performance Results}

In this section, we provide our performance comparisons in order to characterize the effects of CE errors imposed on the achievable performance of the SM scheme. More specifically, we considered three single-RF transmission schemes, i.e., the conventional training-based SM scheme, the joint channel estimation and data detection aided SM scheme as well as the non-coherently detected DSTSK scheme, while employing the optimal reduced-complexity single-stream ML detector for all the schemes. Again, it was also assumed for the coherently-detected SM schemes that the pilot symbols were given by $\mathbf{P}=$ I, which were transmitted at the beginning of each frame of $\tau=200$ SM data symbols, implying that each pilot block contains the total power of $\|\mathbf{P}\|^{2}=M$. We note that the DSTSK scheme did not impose any pilot overhead. According to [11], we assumed the $i$ th-row and $j$ th-column element of $\mathbf{R}_{\mathrm{H}}$ to be $\left[\mathbf{R}_{\mathrm{H}}\right]_{i j}=N \epsilon^{|i-j|}$, where $0 \leq \epsilon<1$ is the channel's correlation coefficient.

Fig. 1 shows the MSE $J_{\mathrm{MMSE}}$ of the estimated channels, which was normalized by $(M \cdot N)$. We also plotted the MSE curves of the multiple-RF pilot transmissions, which allows the elimination of the sparse constraint imposed on the single-RF

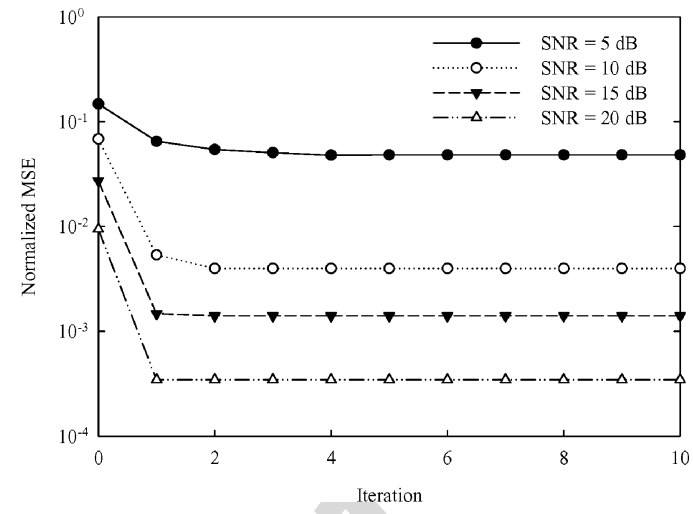

Fig. 2. Normalized MSE of the estimated channels for the single-RF pilot transmissions, where the number of transmit and receive antennas was set to $(M, N)=(8,4)$ for $\mathrm{SNR}=5,10,15$ and $20 \mathrm{~dB}$. The antenna's correlation coefficient was given by $\epsilon=0.8$.

transmitter's pilot symbols. Here, the number of transmit antennas $M$ was varied from $M=2$ to 16 , while the number of receive antennas $N$ was set to $N=4$. Furthermore, the antenna's correlation coefficient was given by $\epsilon=0.8$. It was found in Fig. 1 that in the low-SNR region the SM scheme exhibited a performance penalty in comparison to the classic multiple-RF based MIMO scheme, owing to the sparsity imposed by the SM scheme's pilot symbols. ${ }^{2}$ By contrast, this effect becomes negligible at high SNRs. ${ }^{3}$ Furthermore, upon increasing the number of transmit AEs $M$, the normalized MSE value was deteriorated. This becomes severer for rapidly-fading scenarios exhibiting a high Doppler frequency, where the periodic pilots insertions as well as the CE actions have to be more frequent. Note that for uncorrelated channels of $\epsilon=0$, leading to $\mathbf{R}_{\mathrm{H}}=\mathbf{I}$, the CE MSE curves associated with the single- and multiple-RF pilot transmissions coincided.

Next, in Fig. 2 we investigated the normalized MSE of the estimated channels for the joint $\mathrm{CE}$ and data detection based SM scheme, where the number of transmit and receive AEs was set to $(M, N)=(8,4)$, while considering the SNRs of 5, 10, 15 and $20 \mathrm{~dB}$. The antenna's correlation coefficient was given by $\epsilon=$ 0.8 and the number of iterations $I$ was varied from $I=0$ to 10 . The total power associated with each pilot block $\mathbf{P}$ was $\|\mathbf{P}\|^{2}=$ $M$ both for the single- and multiple-RF scenarios. Considering that the initial MSE values at $I=0$ correspond to the results of the conventional training-based SM scheme of Section III.B, the iterative joint detection scheme of Section III.A was found to significantly improve the CSI-estimation performance upon increasing the number of iterations. Specifically, the MSE value stabilized for a relatively low number of iterations, such as $I \leq$ 3 , regardless of the channel's correlation coefficient $\epsilon$.

In Fig. 3 we plotted the BER curves of the SM schemes, comparing the three CE scenarios of the perfect CSI, the conventional training-based $\mathrm{CE}$ and the joint $\mathrm{CE}$ and data detection

${ }^{2}$ By contrast, practical modems typically employ a powerful channel coding scheme, such as turbo code and low-density parity-check code, typically exhibits a so-called 'BER cliff' in the low SNR regime. Hence, the performance degradation of Fig. 1, which is specific to the single-RF transmitter, may be especially detrimental in such a channel-encoded SM scheme.

${ }^{3}$ In order to expound a little further, it was theoretically shown in [11] that the MSE value of the optimal multiple-RF transmitter converges to that of the $\mathbf{P}=\mathbf{I}$ scenario at high SNRs, which agrees with our analysis. 


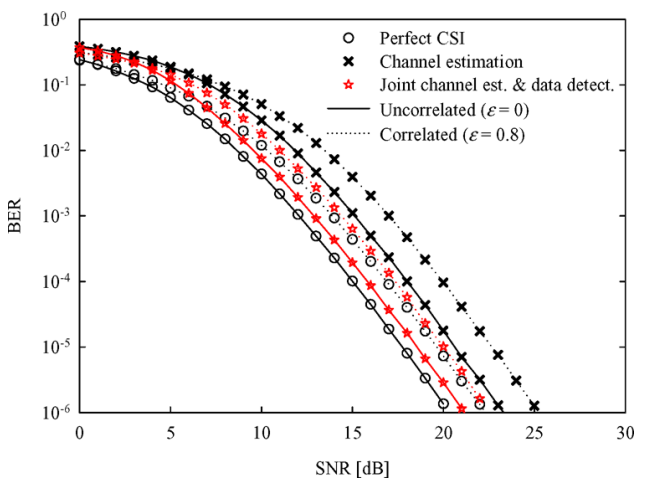

Fig. 3. Comparisons of the SM scheme's achievable BER performance between the three channel-estimation scenarios of the perfect CSI, the conventional training-based channel estimation and the joint channel estimation and data detection scheme, having the system parameters of $(M, N)=(8,4)$, $\mathcal{L}=8$ and $\epsilon=0,0.8$.

scheme. Here, the number of transmit and receive AEs was set to $(M, N)=(8,4)$, while employing $\mathcal{L}=8$-PSK constellations. Hence, the corresponding normalized transmission rate was $R=6 \mathrm{bits} / \mathrm{symbol}$. We also considered both the uncorrelated and correlated scenarios of $\epsilon=0$ and 0.8 . It can be seen in Fig. 3 that our iterative scheme closely approached the perfect-CSI curve in both the correlated and uncorrelated scenarios, while that of the conventional training-based $\mathrm{CE}$ exhibited a 3-dB performance degradation in comparison to other schemes, due to the effect of CE errors.

Finally, Fig. 4 compares the SM scheme's achievable BER performance, where we also considered the non-coherently detected single-RF DSTSK scheme of Section III.B, employing the system parameters of $(M, N, T, Q, \mathcal{L})=(2,2,2,4,4)$, in addition to the three coherently-detected schemes of Fig. 3. Here, the number of transmit and receive AEs was set to $(M, N)=(2,2)$, while employing binary PSK (BPSK) constellations for the three coherently-detected SM schemes. The normalized transmission rate of all the schemes was given by $R=2$ bits/symbol. Furthermore, we considered the correlated scenario of $\epsilon=0.8$. Observe in Fig. 4 that the non-coherent DSTSK scheme outperformed the other three schemes, which was achieved as the benefits of dispensing with $\mathrm{CE}$ and of exploiting the transmit diversity gain. We note that the performance advantage of non-coherent detection becomes more prominent for rapidly-fading scenarios, albeit multiple-symbol differential sphere detection might be required for mitigating the effects of a high Doppler [13].

\section{CONCLUSION}

In this letter, we investigated the effects of training-based CE on the achievable performance of the SM scheme, motivated by the fact that most of the previous studies employed the idealized simplifying assumption of having perfect CSI at the receiver. Our simulation results demonstrated that upon increasing the number of transmit AEs, the transmission power associated with pilot symbols is increased, while the bandwidth efficiency is reduced. Furthermore, especially for the scenario of low SNRs as well as for correlated channels, the single-RF based pilot transmissions suffer from an explicit performance loss in comparison

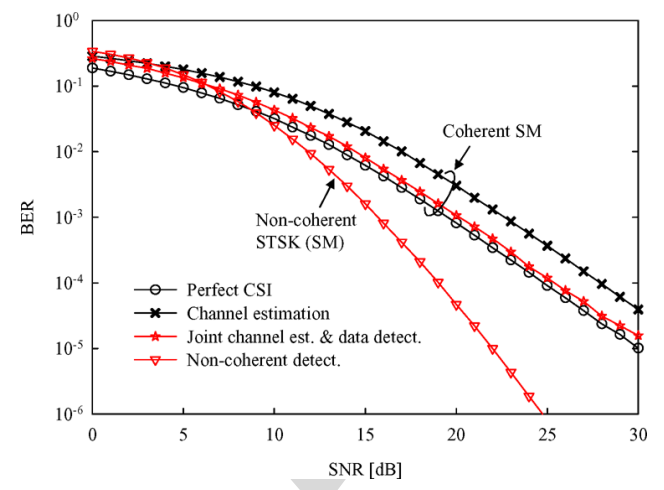

Fig. 4. Comparisons of the SM scheme's achievable BER performance between the three CSI-related scenarios of the perfect CSI, the MMSE CE and the joint $\mathrm{CE}$ and data detection scheme, having the system parameters of $(M, N)=$ $(2,2)$ and $\mathcal{L}=2$ BPSK constellations. We also plotted the BER curve of non-coherently detected single-RF STSK scheme, employing the system parameters of $(M, N, T, Q, \mathcal{L})=(2,2,2,4,4)$.

to those of the classic multiple-RF MIMO arrangements. It was demonstrated that both the proposed joint $\mathrm{CE}$ and data detection aided SM scheme as well as the non-coherently detected STSK scheme are the promising candidates capable of solving these problems, while maintaining the SM scheme's single-RF low-complexity transmitter structure.

\section{REFERENCES}

[1] R. Y. Mesleh, H. Haas, S. Sinanovic, C. Ahn, and S. Yun, "Spatial modulation," IEEE Trans. Veh. Technol., vol. 57, no. 4, pp. 2228-2242, 2008.

[2] J. Jeganathan, A. Ghrayeb, L. Szczecinski, and A. Ceron, "Space shift keying modulation for MIMO channels," IEEE Trans. Wireless Commun., vol. 8, no. 7, pp. 3692-3703, 2009.

[3] M. Di Renzo and H. Haas, "A general framework for performance analysis of space shift keying (SSK) modulation for MISO correlated nakagami-m fading channels," IEEE Trans. Commun., vol. 58, no. 9, pp. 2590-2603, Sept. 2010

[4] M. Di Renzo, H. Haas, and P. M. Grant, "Spatial modulation for multiple-antenna wireless systems: A survey," IEEE Commun. Mag., vol. 49, no. 12, pp. 182-191, 2011.

[5] S. Sugiura, S. Chen, and L. Hanzo, "A universal space-time architecture for multiple-antenna aided systems," IEEE Commun. Surv. Tutorials, vol. 14, no. 2, pp. 401-420, Second quarter 2012.

[6] E. Basar, U. Aygolu, E. Panayirci, and H. Poor, "Performance of spatial modulation in the presence of channel estimation errors," IEEE Commun. Lett., vol. 16, no. 2, pp. 176-179, Feb. 2012.

[7] M. Di Renzo, D. De Leonardis, F. Graziosi, and H. Haas, "Space shift keying (SSK-) MIMO with practical channel estimates," IEEE Trans. Commun., vol. 60, no. 4, pp. 998-1012, Apr. 2012.

[8] S. Sugiura, S. Chen, and L. Hanzo, "Coherent and differential spacetime shift keying: A dispersion matrix approach," IEEE Trans. Commun., vol. 58, no. 11, pp. 3219-3230, Nov. 2010.

[9] S. Chen, S. Sugiura, and L. Hanzo, "Semi-blind joint channel estimation and data detection for space-time shift keying systems," IEEE Signal Process. Lett., vol. 17, no. 12, pp. 993-996, Dec. 2010.

[10] S. Sugiura, C. Xu, S. X. Ng, and L. Hanzo, "Reduced-complexity coherent versus non-coherent QAM-aided space-time shift keying," IEEE Trans. Commun., vol. 59, no. 11, pp. 3090-3101, Nov. 2011.

[11] M. Biguesh and A. B. Gershman, "Training-based MIMO channel estimation: A study of estimator tradeoffs and optimal training signals," IEEE Trans. Signal Process., vol. 54, no. 3, pp. 884-893, 2006.

[12] A. F. Molisch and M. Z. Win, "Mimo systems with antenna selection," IEEE Microw. Mag., vol. 5, no. 1, pp. 46-56, 2004.

[13] L. Lampe, R. Schober, V. Pauli, and C. Windpassinger, "Multiplesymbol differential sphere decoding," IEEE Trans. Commun., vol. 53, no. 12, pp. 1981-1985, 2005. 


\title{
Effects of Channel Estimation on Spatial Modulation
}

\author{
Shinya Sugiura, Senior Member, IEEE, and Lajos Hanzo, Fellow, IEEE
}

\begin{abstract}
In this letter, we investigate the effects of trainingbased channel estimation on the achievable performance of the recent spatial modulation (SM) based multiple-input multiple-output (MIMO) scheme. This is motivated by the fact that the SM transmitter is constituted by a single radio-frequency (RF) branch and multiple antenna elements (AEs), hence simultaneous pilot transmissions from the AEs are impossible, unlike in the classic multiple-RF MIMO transmitters. Our simulation results demonstrate that the SM scheme's BER curve exhibits a performance penalty, while relying on realistic imperfect channel-estimation. In order to combat these limitations, we propose two single-RF arrangements, namely a reduced-complexity joint channel estimation and data detection aided SM scheme as well as a non-coherently detected single-RF space-time shift keying scheme dispensing with channel estimation.
\end{abstract}

Index Terms-Channel estimation, iterative detection, non-coherent detection, single-RF MIMO, space-shift keying, space-time shift keying, spatial modulation, training signals.

\section{INTRODUCTION}

$\mathbf{T}$ HE RECENT encoding concept of spatial modulation (SM) [1]-[3] enables us to exploit the potential of multiple-input multiple-output (MIMO) channels with the aid of a single radio-frequency (RF) transmitter arrangement. More specifically, the SM scheme is characterized by a specific antenna element (AE) activation process, where the index of one out of $M$ transmit AEs conveys additional information. This implies that an increase in the transmission rate is achieved by increasing the number of transmit antennas, rather than by relying on spatial multiplexing activating all the $M$ AEs. Detailed performance comparisons of SM and of the classic MIMO systems have been carried out in [4], [5] and the references therein, while assuming that perfect knowledge of the channel state information (CSI) is available at the receiver.

By contrast, considering that the SM scheme is especially beneficial for a high number of AEs, it is of prime significance to explore the effects of pilot overhead and channel estimation errors. Specifically, the effects of channel estimation

Manuscript received July 12, 2012; revised September 01, 2012; accepted September 11, 2012. Date of publication October 02, 2012; date of current version nulldate. The work of S. Sugiura was supported in part by the Japan Society for the Promotion of Science (JSPS KAKENHI Grant 23760353). The financial support of the RC-UK under the auspices of the India-UK Advanced Technology Centre, as well as of the China-UK 4th generation wireless systems project and that of the European Union under the auspices of the Optimix project, is gratefully acknowledged. The associate editor coordinating the review of this manuscript and approving it for publication was Prof. Azadeh Vosoughi.

S. Sugiura is with Toyota Central R\&D Laboratories, Inc., Nagakute, Aichi, 480-1192, Japan (e-mail: sugiura@ieee.org).

L. Hanzo is with the School of Electronics and Computer Science, University of Southampton, Southampton, SO17 1BJ, U.K. (e-mail: 1h@ecs.soton.ac.uk).

Digital Object Identifier 10.1109/LSP.2012.2221707
(CE) errors on the SM scheme were investigated in [6], [7], by assuming that the $\mathrm{CE}$ error was Gaussian. Furthermore, diverse solutions, dispensing with this idealized simplifying assumption, were proposed in [8], [9], where the SM scheme was extended to support non-coherent detection in [8], while joint channel estimation and data detection was proposed in [9]. Furthermore, since the SM scheme is equipped with a single RF branch and multiple transmit AEs, it is impossible to simultaneously transmit multiple pilot symbols from the antenna elements, unlike in the classic multiple-RF MIMO transmitters. This limitation may impose a performance penalty on the coherently-detected single-RF SM scheme.

Against this background, the novel contributions of this letter are two-fold. We first investigate the performance of the coherently-detected SM scheme, while taking into account the effects of training-based channel estimation at the receiver. Additionally, the single-RF SM scheme's performance penalty is evaluated in terms of the mean-square errors (MSEs) of the estimated channels in comparison to the classic multiple-RF MIMO systems. Furthermore, we propose two single-RF MIMO arrangements, namely a reduced-complexity joint channel estimation and data detection aided SM scheme as well as the non-coherently detected space-time shift keying (STSK) scheme. It is demonstrated by our simulations that the proposed schemes have the potential of combating the above-mentioned CSI-error related limitations.

\section{Signal Model}

\section{A. Spatial Modulation}

Consider the transmitter, relying on $M$ transmit AEs and on classic $\mathcal{L}$-point complex-valued constellations, such as phase-shift keying (PSK) and quadrature-amplitude modulation (QAM). We assumed single-carrier transmissions, according to the related studies [1]-[5]. ${ }^{1}$.

Firstly, a total of $B=\log _{2}(M \cdot \mathcal{L})$ information bits are transmitted per symbol duration, which are then serial-to-parallel (S/P) converted to $B_{1}=\log _{2} M$ and $B_{2}=\log _{2} \mathcal{L}$ bits. Furthermore, the $B_{1}$ bits are used for activating one out of $M$ transmit AEs, while the $B_{2}$ bits are mapped to a PSK/QAM symbol $s_{l}(1 \leq l \leq \mathcal{L})$. Finally, the symbol $s_{l}$ is transmitted from the activated $\mathrm{AE}$, having the index of $m(1 \leq m \leq M)$. Hence, the normalized transmission rate $R$ of the SM scheme is formulated by $R=\log _{2}(M \cdot \mathcal{L})$, which logarithmically increases upon increasing either the number of transmit AEs $M$ or the constellation size $\mathcal{L}$.

\footnotetext{
${ }^{1}$ Typically, the SM scheme based on a single-RF transmitter does not support multi-carrier transmissions. This is because if each sub-carrier employs the antenna activation process in a multi-carrier system, simultaneous symbol transmissions from multiple antenna elements become necessary.
} 
At the $N$-antenna-aided receiver, the received signals $\mathbf{y}=$ $\left[y_{1}, \ldots, y_{N}\right]^{T} \in \mathbb{C}^{N \times 1}$ may be expressed as $\mathbf{y}=s_{l} \mathbf{h}_{m}+\mathbf{v}=$ $\mathbf{H k}_{m, l}+\mathbf{v}$, where we have

$$
\mathbf{k}_{m, l}=[\underbrace{0 \ldots, 0}_{m-1}, s_{l}, \underbrace{0 \ldots, 0}_{M-m}]^{T} .
$$

Here, $\mathbf{H}=\left[\mathbf{h}_{1}, \ldots, \mathbf{h}_{M}\right] \in \mathbb{C}^{N \times M}$ represents the complex-valued channel coefficients, while each noise element of $\mathbf{v} \in \mathbb{C}^{N \times 1}$ is the complex-valued Gaussian random variable obeying $\mathcal{C N}\left(0, N_{0}\right)$, having a zero mean and a variance of $N_{0}$. Based on the received signals $\mathbf{y}$, the estimated channels $\mathbf{H}$ and noise variance $N_{0}$, the transmitted $\mathrm{AE}$ and symbol index set $(m, l)$ is identified with the aid of the optimal reduced-complexity matched-filtering (MF) detector [10].

\section{B. Training-Based CSI Estimation}

In the coherently-detected SM scheme, the training symbols $\mathbf{P} \in \mathbb{C}^{M \times t}$ are periodically transmitted in advance of a frame of data symbols, noting that the integer value of $t$ corresponds to the pilot length. Here, $\mathbf{P}$ is assumed to satisfy the power constraint of $\operatorname{tr}\left[\mathbf{P} \mathbf{P}^{H}\right]=t$. More specifically, the associated received training block $\mathbf{Y}_{t} \in \mathbb{C}^{N \times t}$ may be written as $\mathbf{Y}_{t}=$ $\mathbf{H P}+\mathbf{V}_{t}$, where $\mathbf{V}_{t}$ represents the associated noise components, which are complex-valued Gaussian random variables obeying $\mathcal{C N}\left(0, N_{0}\right)$. Importantly, in the single-RF SM transmitter the pilot matrix $\mathbf{P}$ is sparse, where each column of $\mathbf{P}$ has only a single non-zero element, unlike in the classic multiple-RF MIMO systems.

Let us consider the linear CE, which minimizes the estimation MSE of $\mathbf{H}$. Such a minimum MSE (MMSE) CE may be formulated by

$$
\hat{\mathbf{H}}=\mathbf{Y W}
$$

where $\mathbf{W} \in \mathbb{C}^{t \times M}$ represents the MMSE CE's weights, which are given by [11]

$$
\mathbf{W}=\left(\mathbf{P}^{H} \mathbf{R}_{\mathrm{H}} \mathbf{P}+N_{0} N \mathbf{I}\right)^{-1} \mathbf{P}^{H} \mathbf{R}_{\mathrm{H}}
$$

Here, the channel's correlation matrix is $\mathbf{R}_{\mathrm{H}}=\mathbb{E}\left[\mathbf{H}^{H} \mathbf{H}\right] \in$ $\mathbb{C}^{M \times M}$ and $\mathbb{E}[\cdot]$ is the expectation operation. Furthermore, the associated theoretical CE MSE value $J_{\mathrm{MMSE}}$ is formulated as [11]

$$
\begin{aligned}
J_{\mathrm{MMSE}} & =\operatorname{tr}\left[\mathbb{E}\left\{(\mathbf{H}-\hat{\mathbf{H}})(\mathbf{H}-\hat{\mathbf{H}})^{H}\right\}\right] \\
& =\operatorname{tr}\left[\left(\mathbf{R}_{\mathrm{H}}^{-1}+\frac{1}{N_{0} N} \mathbf{P} \mathbf{P}^{H}\right)^{-1}\right]
\end{aligned}
$$

Assuming for simplicity that the pilot symbols obey $\mathbf{P}=\mathbf{I}$ and that we have an uncorrelated scenario, i.e., $\mathbf{R}_{\mathrm{H}}=\mathbf{I}$, the MSE value of (5) may be simplified to $\bar{J}_{\text {MMSE }}=N_{0} M N / N_{0} N+1$. Furthermore, in the high-SNR limit $\left(N_{0} \rightarrow 0\right)$ the associated CE MSE value per AE becomes $\bar{J}_{\text {MMSE }} /(M \cdot N)=N_{0}$, which may result in a 3-dB performance penalty over the perfect-CSI scenario. Note that the single-RF based transmit antenna selection scheme [12] typically employs a similar pilot-transmission strategy and hence the resultant CE errors at the receiver are also similar. However, the antenna selection scheme requires feedback information from the receiver, while the SM scheme does not.

\section{Proposed Detection Schemes}

In this section, we introduce two beneficial schemes, which have the potential of combating the limitations associated with the above-mentioned pilot overhead and CSI estimation errors.

\section{A. Reduced-Complexity Joint Channel Estimation and Data Detection}

As mentioned in [9], the joint CE and data detection scheme is capable of reducing the effects of CE error, which is achieved without prohibitively increasing the pilot overhead. In order to minimize the performance degradation imposed by $\mathrm{CE}$, while reducing the decoding complexity, here we invoke the reducedcomplexity MF-based data detection combined with the MMSE CE. The detailed iterative process is formulated as follows:

i) Initial channel estimation: The initial values of the channel coefficients $\hat{\mathbf{H}}^{(0)}$ are estimated according to the MMSE criterion of (2), (3), while the iteration index is set to $\iota=0$. Furthermore, we set the received signal block as $\mathbf{Y}=\left[\mathbf{y}_{1}, \ldots, \mathbf{y}_{\tau}\right] \in \mathbb{C}^{N \times \tau}$.

ii) Data detection: A frame of $\tau$ transmitted SM symbols are detected with the aid of the reduced-complexity ML detector of [10], in order to attain the concatenated estimated symbol block of $\mathbf{S}^{(\iota)}=\left[\mathbf{k}_{1}^{(\iota)}, \ldots, \mathbf{k}_{\tau}^{(\iota)}\right] \in \mathbb{C}^{M \times \tau}$.

iii) Channel estimation: The channel estimates $\hat{\mathbf{H}}^{(\iota+1)}$ are updated based on the MMSE criterion of (2), (3), which is expressed as $\hat{\mathbf{H}}^{(\iota+1)}=\mathbf{Y}\left(\mathbf{S}^{(\iota) H} \mathbf{R}_{\mathrm{H}} \mathbf{S}^{(\iota)}+\right.$ $\left.N_{0} N \mathbf{I}\right)^{-1} \mathbf{S}^{(\iota) H} \mathbf{R}_{\mathrm{H}}$

iv) Iteration or termination: If $\left\|\hat{\mathbf{H}}^{(\iota+1)}-\hat{\mathbf{H}}^{(\iota)}\right\|^{2} /(M \cdot N)<$ $\beta$, then terminate the iteration and output the estimated symbols $\mathbf{S}^{(\iota)}$ as well as the associated bits. Otherwise, set $\iota=\iota+1$ and goto 2 ). Furthermore, the termination constant $\beta$ is chosen to be sufficiently low.

Here, we note that the achievable bit-error rate (BER) performance is expected to improve at the expense of an increased decoding complexity, which linearly increases with the number of iterations.

\section{B. Non-Coherently Detected Single-RF STSK Scheme}

Additionally, in order to allow the single-RF SM arrangement to remain unaffected by CSI estimation errors, non-coherent detection is invoked in this section. To be more specific, as an extension of the SM scheme, the differentially-encoded space-time shift keying (DSTSK) scheme [8] was developed, which is capable of attaining a transmit diversity gain, while invoking non-coherent detection. Although the DSTSK transmitter was basically assumed to have multiple RF branches, in this letter we relax this constraint, by assuming that the DSTSK uses a single-RF transmitter. Let us assume that our single-RF transmitter has $M$ transmit AEs and $Q$ unitary dispersion matrices $\mathbf{B}_{q} \in \mathbb{C}^{M \times M}(q=1, \ldots, Q)$ [10]. Furthermore, we assume that each dispersion matrix has a sparse structure, where each column of $\mathbf{B}_{q}$ has a single non-zero unit-norm element. 


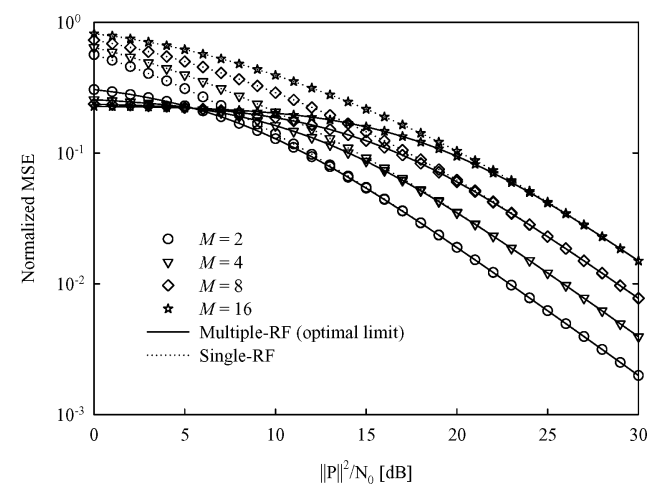

Fig. 1. Normalized MSE of the estimated channels regarding both the singleand multiple-RF pilot transmissions, where the number of transmit antennas $M$ was varied from $M=2$ to 16 , while the number of receive antennas $N$ was set to $N=4$. The antenna's correlation coefficient was given by $\epsilon=0.8$.

Similar to the SM scheme of Section II.A, the $\bar{B}=$ $\log _{2}(Q \cdot \mathcal{L})$ information bits per block are firstly $\mathrm{S} / \mathrm{P}$ converted to $\bar{B}_{1}=\log _{2} Q$ and $\bar{B}_{2}=\log _{2} \mathcal{L}$ bits, which are then signaled by activating one out of $Q$ dispersion matrices $\mathbf{B}_{q}(n)$ and by a PSK/QAM symbol $s_{l}(n)$, respectively. Here, $n$ denotes the block index. Finally, the differentially-encoded symbols are generated based on two successive received-signal blocks as follows: $\overline{\mathbf{S}}(n)=s_{l}(n) /\left|s_{l}(n-1)\right| \overline{\mathbf{S}}(n-1) \mathbf{B}_{q}(n) \in \mathbb{C}^{M \times M}$, where $\overline{\mathbf{S}}(n)$ also has a sparse matrix structure, similar to $\mathbf{B}_{q}$. This ensures that our scheme transmits a single symbol per transmission interval. The signal block received during the $n$th block interval is written as $\overline{\mathbf{Y}}(n)=\mathbf{H} \overline{\mathbf{S}}(n)+\overline{\mathbf{V}}(n) \in \mathbb{C}^{M \times M}$. Finally, the transmitted information bits are estimated without resorting to any channel estimation [10], assuming that the channel matrix $\mathbf{H}$ remains unchanged over the two block intervals. Naturally, the achievable BER performance of the single-RF DSTSK scheme proposed in this section is upper-bounded by that of the full-RF DSTSK scheme of [8].

\section{Performance Results}

In this section, we provide our performance comparisons in order to characterize the effects of CE errors imposed on the achievable performance of the SM scheme. More specifically, we considered three single-RF transmission schemes, i.e., the conventional training-based SM scheme, the joint channel estimation and data detection aided SM scheme as well as the non-coherently detected DSTSK scheme, while employing the optimal reduced-complexity single-stream ML detector for all the schemes. Again, it was also assumed for the coherently-detected SM schemes that the pilot symbols were given by $\mathbf{P}=$ $\mathbf{I}$, which were transmitted at the beginning of each frame of $\tau=200$ SM data symbols, implying that each pilot block contains the total power of $\|\mathbf{P}\|^{2}=M$. We note that the DSTSK scheme did not impose any pilot overhead. According to [11], we assumed the $i$ th-row and $j$ th-column element of $\mathbf{R}_{\mathrm{H}}$ to be $\left[\mathbf{R}_{\mathrm{H}}\right]_{i j}=N \epsilon^{|i-j|}$, where $0 \leq \epsilon<1$ is the channel's correlation coefficient.

Fig. 1 shows the MSE $J_{\mathrm{MMSE}}$ of the estimated channels, which was normalized by $(M \cdot N)$. We also plotted the MSE curves of the multiple-RF pilot transmissions, which allows the elimination of the sparse constraint imposed on the single-RF

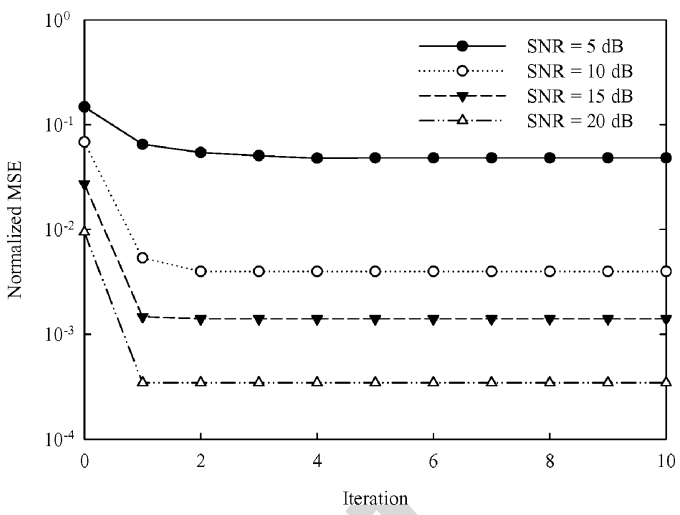

Fig. 2. Normalized MSE of the estimated channels for the single-RF pilot transmissions, where the number of transmit and receive antennas was set to $(M, N)=(8,4)$ for SNR $=5,10,15$ and $20 \mathrm{~dB}$. The antenna's correlation coefficient was given by $\epsilon=0.8$.

transmitter's pilot symbols. Here, the number of transmit antennas $M$ was varied from $M=2$ to 16 , while the number of receive antennas $N$ was set to $N=4$. Furthermore, the antenna's correlation coefficient was given by $\epsilon=0.8$. It was found in Fig. 1 that in the low-SNR region the SM scheme exhibited a performance penalty in comparison to the classic multiple-RF based MIMO scheme, owing to the sparsity imposed by the SM scheme's pilot symbols. ${ }^{2}$ By contrast, this effect becomes negligible at high SNRs. ${ }^{3}$ Furthermore, upon increasing the number of transmit AEs $M$, the normalized MSE value was deteriorated. This becomes severer for rapidly-fading scenarios exhibiting a high Doppler frequency, where the periodic pilots insertions as well as the $\mathrm{CE}$ actions have to be more frequent. Note that for uncorrelated channels of $\epsilon=0$, leading to $\mathbf{R}_{\mathrm{H}}=\mathbf{I}$, the CE MSE curves associated with the single- and multiple-RF pilot transmissions coincided.

Next, in Fig. 2 we investigated the normalized MSE of the estimated channels for the joint $\mathrm{CE}$ and data detection based SM scheme, where the number of transmit and receive AEs was set to $(M, N)=(8,4)$, while considering the SNRs of 5, 10, 15 and $20 \mathrm{~dB}$. The antenna's correlation coefficient was given by $\epsilon=$ 0.8 and the number of iterations $I$ was varied from $I=0$ to 10 . The total power associated with each pilot block $\mathbf{P}$ was $\|\mathbf{P}\|^{2}=$ $M$ both for the single- and multiple-RF scenarios. Considering that the initial MSE values at $I=0$ correspond to the results of the conventional training-based SM scheme of Section III.B, the iterative joint detection scheme of Section III.A was found to significantly improve the CSI-estimation performance upon increasing the number of iterations. Specifically, the MSE value stabilized for a relatively low number of iterations, such as $I \leq$ 3 , regardless of the channel's correlation coefficient $\epsilon$.

In Fig. 3 we plotted the BER curves of the SM schemes, comparing the three CE scenarios of the perfect CSI, the conventional training-based $\mathrm{CE}$ and the joint $\mathrm{CE}$ and data detection

\footnotetext{
${ }^{2}$ By contrast, practical modems typically employ a powerful channel coding scheme, such as turbo code and low-density parity-check code, typically exhibits a so-called 'BER cliff' in the low SNR regime. Hence, the performance degradation of Fig. 1, which is specific to the single-RF transmitter, may be especially detrimental in such a channel-encoded SM scheme.

${ }^{3}$ In order to expound a little further, it was theoretically shown in [11] that the MSE value of the optimal multiple-RF transmitter converges to that of the $\mathbf{P}=\mathbf{I}$ scenario at high SNRs, which agrees with our analysis.
} 


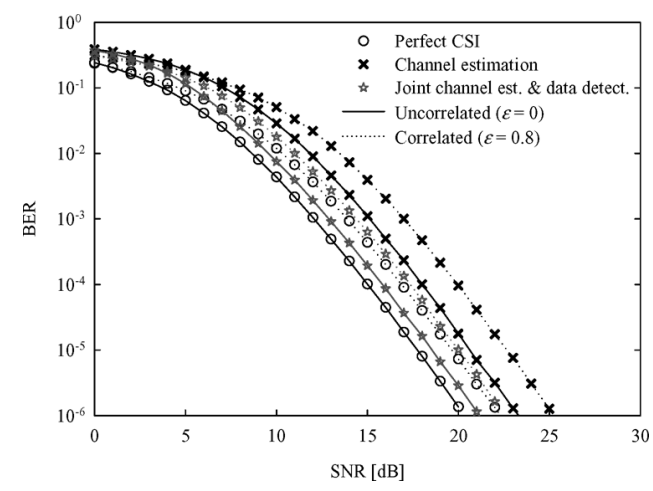

Fig. 3. Comparisons of the SM scheme's achievable BER performance between the three channel-estimation scenarios of the perfect CSI, the conventional training-based channel estimation and the joint channel estimation and data detection scheme, having the system parameters of $(M, N)=(8,4)$, $\mathcal{L}=8$ and $\epsilon=0,0.8$.

scheme. Here, the number of transmit and receive AEs was set to $(M, N)=(8,4)$, while employing $\mathcal{L}=8$-PSK constellations. Hence, the corresponding normalized transmission rate was $R=6$ bits/symbol. We also considered both the uncorrelated and correlated scenarios of $\epsilon=0$ and 0.8. It can be seen in Fig. 3 that our iterative scheme closely approached the perfect-CSI curve in both the correlated and uncorrelated scenarios, while that of the conventional training-based $\mathrm{CE}$ exhibited a 3 - $\mathrm{dB}$ performance degradation in comparison to other schemes, due to the effect of CE errors.

Finally, Fig. 4 compares the SM scheme's achievable BER performance, where we also considered the non-coherently detected single-RF DSTSK scheme of Section III.B, employing the system parameters of $(M, N, T, Q, \mathcal{L})=(2,2,2,4,4)$, in addition to the three coherently-detected schemes of Fig. 3. Here, the number of transmit and receive AEs was set to $(M, N)=(2,2)$, while employing binary PSK (BPSK) constellations for the three coherently-detected SM schemes. The normalized transmission rate of all the schemes was given by $R=2$ bits/symbol. Furthermore, we considered the correlated scenario of $\epsilon=0.8$. Observe in Fig. 4 that the non-coherent DSTSK scheme outperformed the other three schemes, which was achieved as the benefits of dispensing with $\mathrm{CE}$ and of exploiting the transmit diversity gain. We note that the performance advantage of non-coherent detection becomes more prominent for rapidly-fading scenarios, albeit multiple-symbol differential sphere detection might be required for mitigating the effects of a high Doppler [13].

\section{CONCLUSION}

In this letter, we investigated the effects of training-based CE on the achievable performance of the SM scheme, motivated by the fact that most of the previous studies employed the idealized simplifying assumption of having perfect CSI at the receiver. Our simulation results demonstrated that upon increasing the number of transmit AEs, the transmission power associated with pilot symbols is increased, while the bandwidth efficiency is reduced. Furthermore, especially for the scenario of low SNRs as well as for correlated channels, the single-RF based pilot transmissions suffer from an explicit performance loss in comparison

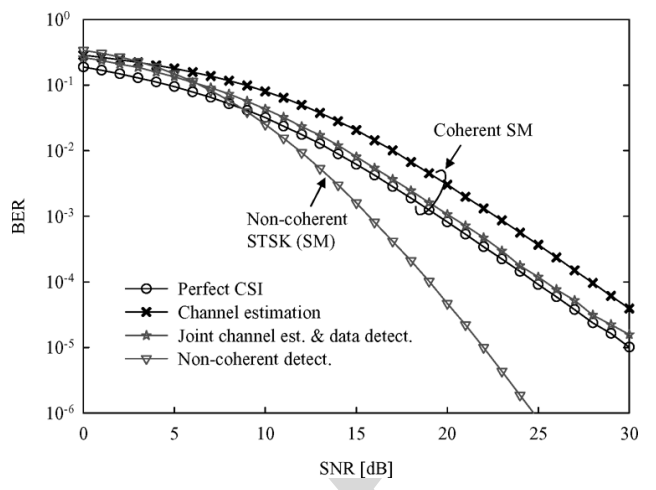

Fig. 4. Comparisons of the SM scheme's achievable BER performance between the three CSI-related scenarios of the perfect CSI, the MMSE CE and the joint $\mathrm{CE}$ and data detection scheme, having the system parameters of $(M, N)=$ $(2,2)$ and $\mathcal{L}=2$ BPSK constellations. We also plotted the BER curve of non-coherently detected single-RF STSK scheme, employing the system parameters of $(M, N, T, Q, \mathcal{L})=(2,2,2,4,4)$.

to those of the classic multiple-RF MIMO arrangements. It was demonstrated that both the proposed joint $\mathrm{CE}$ and data detection aided SM scheme as well as the non-coherently detected STSK scheme are the promising candidates capable of solving these problems, while maintaining the SM scheme's single-RF low-complexity transmitter structure.

\section{REFERENCES}

[1] R. Y. Mesleh, H. Haas, S. Sinanovic, C. Ahn, and S. Yun, "Spatial modulation," IEEE Trans. Veh. Technol., vol. 57, no. 4, pp. 2228-2242, 2008.

[2] J. Jeganathan, A. Ghrayeb, L. Szczecinski, and A. Ceron, "Space shift keying modulation for MIMO channels," IEEE Trans. Wireless Commun., vol. 8, no. 7, pp. 3692-3703, 2009.

[3] M. Di Renzo and H. Haas, "A general framework for performance analysis of space shift keying (SSK) modulation for MISO correlated nakagami-m fading channels," IEEE Trans. Commun., vol. 58, no. 9, pp. 2590-2603, Sept. 2010

[4] M. Di Renzo, H. Haas, and P. M. Grant, "Spatial modulation for multiple-antenna wireless systems: A survey," IEEE Commun. Mag., vol. 49, no. 12, pp. 182-191, 2011.

[5] S. Sugiura, S. Chen, and L. Hanzo, "A universal space-time architecture for multiple-antenna aided systems," IEEE Commun. Surv. Tutorials, vol. 14, no. 2, pp. 401-420, Second quarter 2012.

[6] E. Basar, U. Aygolu, E. Panayirci, and H. Poor, "Performance of spatial modulation in the presence of channel estimation errors," IEEE Commun. Lett., vol. 16, no. 2, pp. 176-179, Feb. 2012.

[7] M. Di Renzo, D. De Leonardis, F. Graziosi, and H. Haas, "Space shift keying (SSK-) MIMO with practical channel estimates," IEEE Trans. Commun., vol. 60, no. 4, pp. 998-1012, Apr. 2012.

[8] S. Sugiura, S. Chen, and L. Hanzo, "Coherent and differential spacetime shift keying: A dispersion matrix approach," IEEE Trans. Commun., vol. 58, no. 11, pp. 3219-3230, Nov. 2010.

[9] S. Chen, S. Sugiura, and L. Hanzo, "Semi-blind joint channel estimation and data detection for space-time shift keying systems," IEEE Signal Process. Lett., vol. 17, no. 12, pp. 993-996, Dec. 2010.

[10] S. Sugiura, C. Xu, S. X. Ng, and L. Hanzo, "Reduced-complexity coherent versus non-coherent QAM-aided space-time shift keying," IEEE Trans. Commun., vol. 59, no. 11, pp. 3090-3101, Nov. 2011.

[11] M. Biguesh and A. B. Gershman, "Training-based MIMO channel estimation: A study of estimator tradeoffs and optimal training signals," IEEE Trans. Signal Process., vol. 54, no. 3, pp. 884-893, 2006.

[12] A. F. Molisch and M. Z. Win, "Mimo systems with antenna selection," IEEE Microw. Mag., vol. 5, no. 1, pp. 46-56, 2004.

[13] L. Lampe, R. Schober, V. Pauli, and C. Windpassinger, "Multiplesymbol differential sphere decoding," IEEE Trans. Commun., vol. 53, no. 12, pp. 1981-1985, 2005. 